\title{
La integración económica y la competitividad en el sector eléctrico: análisis de casos en América Latina
}

Alfonso Brown del Rivero* y Jordi Bacaria Colom**

Resumen: Se analiza la relación entre los procesos de reforma y liberalización eléctrica emprendidos a partir de los años ochenta, y su conexión con diferentes modelos de integración latinoamericana. Se contrasta la configuración de los mercados y las experiencias de reforma en Argentina, Chile y México con la de España. La conclusión es que los procesos de reforma y liberalización están íntimamente correlacionados con la integración económica, y para que ésta sea más profunda se requiere la existencia de instituciones supranacionales que dicten políticas de competencia y libre circulación de capitales, al mismo tiempo que vigilen su cumplimiento.

Palabras Clave: Integración, América Latina, Electricidad, Reforma, Competencia, Instituciones supranacionales.

ABSTRACT: In this article we analyze the relation between the processes of reform and liberalization electric power supply industry initiated in the eighties and its relationship with different models of Latin American integration. We also contrast the configuration of markets and reform experiences in Argentina, Chile and Mexico to Spain. The conclusion is that the processes of reform and liberalization are closely correlated with economic integration, and for it to be deeper, requires the existence of supranational institutions that dictate policies of competition and free movement of capitals at the same time they monitor its compliance.

Key words: Integration, Latin America, Electricity, Reform, Competition, Supranational institutions.

Departamento de Economía del Instituto Tecnológico de Monterrey, Campus Ciudad de México (abrown@itesm.mx).

** Universidad Autónoma de Barcelona (Jordi.Bacaria@uab.cat). 


\section{INTRODUCCIÓN}

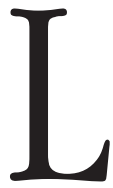

a integración de América Latina y el Caribe ha tenido un progreso muy importante durante las dos últimas décadas, a pesar de los conflictos y dificultades para resolver los obstáculos que se presentan de manera natural en estos procesos. También han surgido esquemas de integración basados en una lógica distinta.

Dos factores han sido los causantes principales del avance en el proceso de integración de la región: 1) una convergencia de regímenes democráticos en la mayor parte de los países, y 2) una coincidencia de políticas económicas dirigidas a promover la competitividad de los mercados, la liberalización económica, la desregulación y la apertura en las relaciones comerciales con el exterior. La apertura unilateral (regionalismo abierto) representó al comienzo la vía escogida para capitalizar los beneficios de las reformas emprendidas. Una vez agotada esta estrategia, la integración regional fue el camino natural elegido. No obstante, la crisis asiática y los ajustes cambiarios de varios países latinoamericanos hicieron que se perdiera el impulso inicial. Ya en la presente década surgieron duras críticas al proceso de reformas iniciado a finales de los ochenta y principios de los noventa y junto con ello nuevas propuestas de integración regional. ${ }^{1}$

La integración de la región ha tomado diferentes caminos. En el primero se ubican la Comunidad Andina (CAN), la Comunidad del Caribe (Caricom, por sus siglas en inglés), el Mercado Común Centroamericano (MCCA) y el Mercado Común del Sur (Mercosur). En el segundo están las negociaciones comerciales bilaterales, ya sea en forma individual o como bloque. Por último, en la tercera vía se encuentran los acuerdos suscritos con países fuera de la región. Chile y México son países que se han destacado por su activismo en la segunda y en la tercera opciones.

\footnotetext{
${ }^{1}$ Sebastián Sáez, La integración en busca de un modelo: los problemas de convergencia en América Latina y el Caribe, CEPal, Serie Comercio Internacional, 88, Santiago de Chile, 2008.
} 


\section{Reforma ELÉCTRICA EN AmÉRICA LaTINA}

Uno de los sectores en el que puede apreciarse mejor los beneficios de la simbiosis entre reforma competitiva de mercado e integración económica regional es el eléctrico. La mayoría de los países de la región comenzó una liberalización gradual de sus economías a mediados de los ochenta. Las reformas que se emprendieron surgieron en un contexto caracterizado por apremiantes restricciones fiscales y de balanza de pagos. Los procesos de liberalización y reforma fueron forzados por las circunstancias, dado que los gobiernos se vieron urgidos a sanear sus finanzas. Esto permitió la apertura de la participación privada en nuevas actividades y la privatización de empresas públicas. Por su parte, la liberalización comercial produjo importantes beneficios, tanto por la reducción arancelaria como por los efectos dinámicos que propiciaron un auge en la inversión, así como una mayor competencia. No obstante, varios de estos procesos de reforma y liberalización comercial quedaron incompletos y en algunos países subsisten, todavía, importantes rezagos que se traducen en ineficiencias y altos costos para toda la economía.

Antes de que se llevaran a cabo las reformas, todos los sistemas eléctricos de los países de la región estaban dominados por empresas públicas o tenían presencia exclusiva de ellas. Las empresas estaban integradas verticalmente y el funcionamiento de los sistemas eléctricos estaba bajo el control del Estado. Las tarifas y las decisiones de inversión se regían por criterios ajenos al mercado, intentaban promover el desarrollo pero con mucha frecuencia prevalecían intereses políticos de corto plazo.

Los procesos de reforma se orientaron, en la mayoría de los países, a introducir mecanismos de mercado por medio de la privatización de empresas públicas y la apertura de algunas actividades al sector privado, así como la desregulación y adopción de un nuevo marco regulatorio. Como puede apreciarse en la Gráfica 1, la tendencia ha sido la apertura del mercado y la privatización de la industria, desde el cuadrante (a) hacia el cuadrante (h). Los casos extremos son los de Argentina, Bolivia, Guatemala, Chile, Perú y El Salvador, por un lado, y el de México y Cuba, por el otro. Incluso en México, gracias a la reforma de la Ley del Servicio Público de Electricidad y Energía en 1992, se per- 
mitió, aunque bajo condiciones muy limitadas, la participación privada en la generación de electricidad.

Gráfica 1

Procesos de reforma de los sistemas eléctricos en América Latina

\begin{tabular}{|c|c|c|c|c|c|}
\hline \multirow{2}{*}{$\begin{array}{l}\text { Propiedad } \\
\text { privada }\end{array}$} & $\begin{array}{l}\text { Segmentación } \\
\text { vertical con } \\
\text { incompatibilidad } \\
\text { de funciones }\end{array}$ & & & \multirow[t]{2}{*}{ 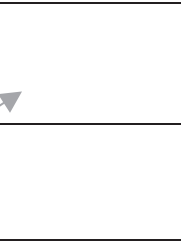 } & $\begin{array}{r}\text { (h) } \\
\text { Argentina }(* * *) \\
\text { Bolivia }(* * *) \\
\text { Guatemala }(* * *)\end{array}$ \\
\hline & $\begin{array}{l}\text { Integración } \\
\text { vertical } \\
\text { permitida }\end{array}$ & & (g) & & $\begin{array}{r}\text { (f) } \\
\text { Chile }(* *) \\
\text { Perú }(* *) \\
\text { El Salvador }(* *)\end{array}$ \\
\hline \multicolumn{2}{|c|}{$\begin{array}{l}\text { Propiedad mixta } \\
\text { o participación } \\
\text { privada parcial }\end{array}$} & & $\begin{array}{r}\text { (d) } \\
\text { Venezuela } \\
\text { Brasil } \\
\text { Costa Rica } \\
\text { Ecuador }\end{array}$ & & $\begin{array}{l}(\mathrm{e})\left({ }^{*}\right) \\
\text { Colombia }(* *) \\
\text { Panamá }\left({ }^{* *}\right)\end{array}$ \\
\hline \multirow[t]{2}{*}{$\begin{array}{l}\text { Propiedad } \\
\text { exclusiva }\end{array}$} & & $\begin{array}{l}\text { (a) } \\
\text { México } \\
\text { Cuba }\end{array}$ & $\begin{array}{r}\text { Uruguay } \\
\text { Paraguay } \\
\text { Haití }\end{array}$ & $\begin{array}{r}\text { (c) } \\
\text { Surinam } \\
\text { Guyana } \\
\text { Nicaragua } \\
\text { Jamaica } \\
\text { Honduras } \\
\text { Trinidad y Tobago } \\
\text { República } \\
\text { Dominicana }\end{array}$ & \\
\hline & & $\begin{array}{l}\text { Control } \\
\text { estatal }\end{array}$ & $\begin{array}{l}\text { Integrada } \\
\text { regulada }\end{array}$ & $\begin{array}{l}\text { Comprador } \\
\text { único }\end{array}$ & $\begin{array}{c}\text { Mercado } \\
\text { Abierto }\end{array}$ \\
\hline
\end{tabular}

(*) Con o sin desintegración vertical (incompatibilidad de funciones) estricta

(**) Con alto grado de concentración técnica y económica

(**) Con concentración técnica y económica intermedia o baja

FuENTE: CEPAL, OLADE, GTZ, "Energía y desarrollo sustentable en América Latina y el Caribe: guía para la formulación de políticas energéticas", en Cuadernos de la CEPAL, núm. 89, Santiago de Chile, diciembre de 2003, p. 84.

Chile fue el pionero, no sólo en América Latina, sino en el mundo, en emprender una profunda reforma de su sector eléctrico en 1978. En ese año se inició una amplia reestructuración de su industria, mediante la separación vertical de las actividades que realizaban las empresas eléctricas y quedaron divididas para garantizar una mayor eficiencia. Se crearon nuevas empresas privadas y se introdujeron reglas distintas de funcionamiento con la supervisión de la Comi- 
sión Nacional de la Energía, el organismo regulador. Se introdujo también competencia en los mercados eléctricos, al permitir que los grandes usuarios pudieran escoger su proveedor y negociar libremente los precios. Posteriormente, se fijaron mecanismos de mercado específicos para determinar los despachos de los generadores y el precio de la electricidad al mayoreo, con lo cual se propició la competencia entre ellos.

La integración energética y en particular la eléctrica entre los países latinoamericanos se limitó inicialmente a grandes obras, para aprovechar recursos compartidos o de interconexión eléctrica con un escaso comercio entre los países. Con los procesos de reforma no sólo cambió la configuración de los mercados, sino también las posibilidades de integración. El grado de integración está de modo directo correlacionado con el alcance y la profundidad de las reformas llevadas a cabo. Así, la integración tiene mayor impulso en las subregiones, donde se han realizado los procesos de reforma más ambiciosos y en los que, gracias a la apertura, el sector privado tiene una participación más activa. No obstante los principales logros se han alcanzado en la liberalización del comercio y no en aspectos de cooperación y complementación productiva. ${ }^{2}$

En otro contexto de integración regional, España es un buen ejemplo de los efectos positivos que generan la liberalización de los mercados y la integración económica sobre la competitividad y el desarrollo. Dicho país fue capaz de liberalizar sus mercados y modernizar su estructura institucional en un periodo de tiempo relativamente corto, lo cual no puede entenderse al margen del proceso de adhesión y convergencia con la Comunidad Económica Europea (CEE) y la Unión Europea (UE). Ambos procesos se reforzaron mutuamente no sólo por el impacto de las políticas e instituciones comunitarias, sino también por el efecto de la competencia externa como catalizador de reformas internas. En algunos sectores, como el eléctrico, España ha sido pionera en introducir reformas dentro de la UE.

El Tratado de Adhesión a la ceE y el Programa de Mercado Único, puestos en marcha en 1985, permitieron que España emprendiera un programa ineludible de liberalización de sus mercados y minimizara sus costos políticos, ya que no fue necesario discutir y negociar cada una de las partes del mismo, sino el

2 Véase CEPAL, OLADE, GTZ, op. cit. 
conjunto de condiciones de acceso y la consecuente adaptación interna conforme a las instituciones supranacionales. ${ }^{3}$

La liberalización comercial había sometido previamente a diversos sectores industriales a una intensa competencia y era indispensable llevar a cabo una liberalización, acorde con los mercados de mayor incidencia sobre los costos y la rentabilidad de las industrias expuestas a la competencia externa. De esta forma, se liberalizaron las industrias eléctrica, petrolera y de gas.

Debido a lo anterior y a las similitudes con América Latina, se ha escogido a España para contrastar el desempeño de su sector eléctrico con el de Argentina, Chile y México como casos representativos de la región, y de fases diferentes en el proceso de reforma expuesto en la Gráfica 1. La utilidad de este análisis radica en que a pesar de las numerosas semejanzas entre ellos, también hay rasgos diferentes que explican la existencia de condiciones más competitivas. Por supuesto esto ha obedecido a varios factores, pero en el caso de España la adopción de políticas de liberalización de los mercados, las reformas institucionales y la integración a la ue fueron factores que contribuyeron en forma decisiva. Por otra parte, cada país está inmerso en modelos de integración económica distintos y este factor puede ser determinante del alcance y contenido de los procesos de reforma del sector eléctrico, así como del desempeño del mismo.

Tendríamos que agregar otra dimensión en la Gráfica 1 para poder representar a España. Esa dimensión es la existencia de instituciones comunitarias que fijan el rumbo en materia de competencia y regulación específica de la industria eléctrica (Comisión Europea), así como la encargada de vigilar la adopción y el cumplimiento del derecho comunitario (Tribunal de Justicia de la Unión Europea). Estas instituciones supranacionales desempeñan un papel particularmente relevante para impulsar y destrabar los procesos nacionales de reforma. Como demostraron Olson ${ }^{4}$ y Stigler, ${ }^{5}$ grupos sociales con intereses específicos

${ }^{3}$ Miguel A. Fernández Ordóñez, La competencia, Madrid, Alianza Editorial, 2000.

${ }^{4}$ Mancur Olson, The Logic of Collective Action: Public Goods and the Theory of Groups, Cambridge, Massachusetts, Harvard University Press, 1965.

${ }^{5}$ George Stigler, "The Theory of Economic Regulation", en Bell's Journal of Economics and Ma nagement Science, primavera, 1971, pp. 3-21. 
y bajos costos de organización colectiva actúan para proteger sus propios intereses, y obtener transferencias de riqueza a su favor mediante diversas regulaciones o restricciones a la competencia o al libre comercio. La integración regional en sus fases más avanzadas promueve la creación de instituciones supranacionales que sirven de anclaje e impulso a las reformas de mercado ${ }^{6}$ y permiten, de esa forma, romper el poder que ejercen determinados grupos de presión nacionales que compiten por preservar o acrecentar sus rentas a partir de una determinada estructura institucional. ${ }^{7}$

La magnitud y composición de la industria eléctrica de cada uno de los países antes mencionados puede apreciarse en el Cuadro 1, en el que se aprecia que los mayores mercados son México y España de la misma forma que los más eficientes, en términos del porcentaje de pérdidas en la transmisión y distribución de electricidad son España y Chile, mientras que Argentina y México presentan condiciones similares.

\section{Cuadro 1}

\section{Generación de electricidad por fuente de energía} 2007

\begin{tabular}{|l|c|c|c|c|}
\hline & Argentina & Chile & México & España \\
\hline Pérdidas en la transmisión y distribución & $16 \%$ & $8 \%$ & $16 \%$ & $5 \%$ \\
\hline Consumo per cápita (KWh) & 2659 & 3318 & 2036 & 6296 \\
\hline Carbón & $2 \%$ & $23 \%$ & $12 \%$ & $25 \%$ \\
\hline Petróleo & $9 \%$ & $25 \%$ & $20 \%$ & $6 \%$ \\
\hline Gas & $54 \%$ & $8 \%$ & $49 \%$ & $31 \%$ \\
\hline Nuclear & $6 \%$ & $0 \%$ & $4 \%$ & $18 \%$ \\
\hline Hidroeléctrica & $27 \%$ & $40 \%$ & $11 \%$ & $9 \%$ \\
\hline Otros renovables & $2 \%$ & $4 \%$ & $4 \%$ & $11 \%$ \\
\hline Producción total $(\mathrm{GWh})$ & 115081 & 58509 & 257455 & 300248 \\
\hline
\end{tabular}

FuENTE: Banco Mundial, World Development Indicators.

La Gráfica 2 muestra la evolución de la generación neta en cada país y en ella se aprecia de manera más clara el tamaño de los respectivos mercados.

${ }^{6}$ Antón Costas, "Economía política de la regulación y la desregulación en España", en J. C. Jiménez [ed.], La economía española ante el nuevo siglo, Madrid, Cívitas, 2001, pp. 39-64.

${ }^{7}$ Mancur Olson, The Rise and Decline of Nations: Economic Growth, Stagflation and Social Rigidities, New Haven, Yale University Press, 1982. 
Gráfica 2

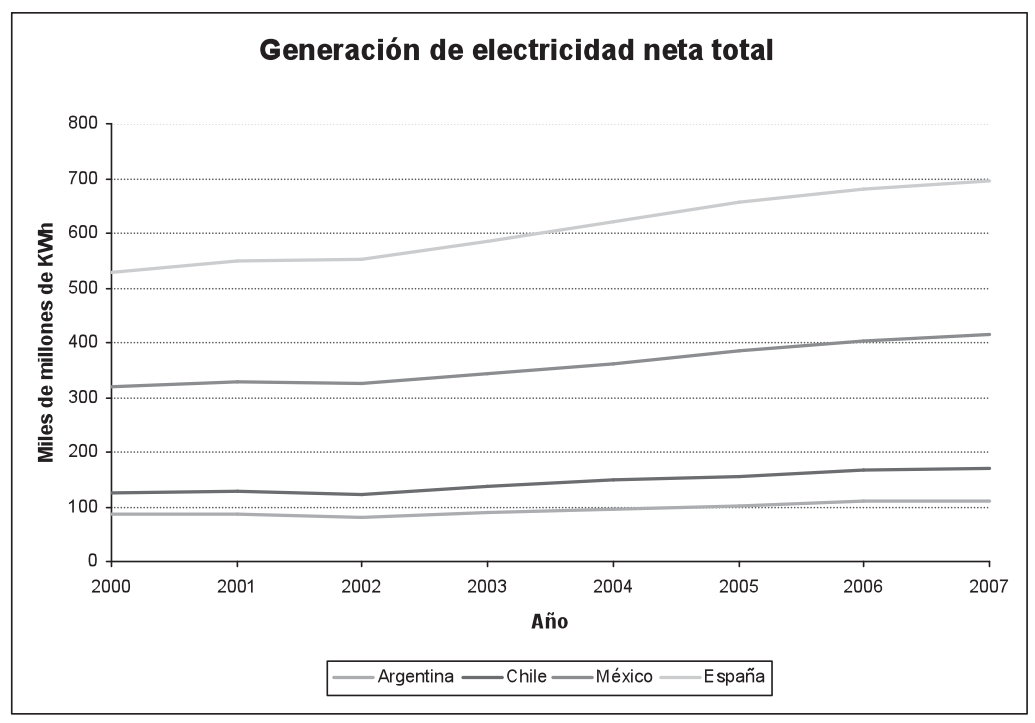

Elaboración propia con base en información del Banco Mundial, World Development Indicators.

En la industria eléctrica el marco institucional desempeña un papel crucial. Las características y limitaciones físicas de la producción y suministro de electricidad demandan un mayor grado de centralización y coordinación en la toma de decisiones, que las requeridas en otras industrias. La adopción de mecanismos de mercado representa un cambio drástico en la organización industrial, y ofrece importantes enseñanzas acerca de las fuerzas que determinan y configuran las condiciones de competencia. Abarca aspectos relacionados con la minimización de los costos de transacción que se espera alcanzar con las reformas de la industria, así como la protección y garantía de los derechos de propiedad y la corrección de fallas de mercado, por medio de instituciones y políticas que promuevan la competencia. Lo anterior comprende una compleja negociación de intereses y factores difíciles de medir. El estudio de casos comparativos es una metodología apropiada de análisis para este tipo de investigaciones. La selección se ha 
basado en el criterio de casos similares. Los casos escogidos muestran variación en los resultados, pero contienen elementos comunes en otras dimensiones. Los países seleccionados, Argentina, Chile, España y México, forman parte de procesos de integración económica distintos, muestran resultados diferentes en el desempeño de sus sectores eléctricos y, al mismo tiempo, comparten rasgos culturales, históricos e institucionales comunes.

\section{ANÁLISIS EMPÍRICO}

Buena parte de los estudios empíricos sobre los resultados de la liberalización del sector eléctrico se centran en comparaciones simples, entre el estado previo y posterior a las reformas con base en limitados indicadores sobre el desempeño operativo y financiero. Por esa razón se considera necesario examinar, de manera conjunta, el efecto de diversos factores institucionales sobre variables de desempeño. Los efectos de la estructura de propiedad, la desagregación de la industria y la regulación deben ser tomados en cuenta en forma conjunta. Dado que el propósito inicial es distinguir el efecto diferencial que ocasiona un conjunto de variables institucionales sobre determinadas variables de desempeño de un grupo de países, se considera que las técnicas más apropiadas son el Análisis de Varianza y el Análisis Multivariante de Varianza (Anova y Manova por sus siglas en inglés, respectivamente).

Las reformas eléctricas involucran actividades multifacéticas que interactúan con una variedad de factores y de impactos. El proceso abarca, generalmente, un conjunto de medidas concretas o medidas basadas en un modelo específico de reforma. En un nivel estas medidas comprenden cambios estructurales y organizacionales en la industria, y en otro requieren de ciertos arreglos institucionales apropiados como en la legislación y la creación de nuevos organismos o mayores facultades en los ya existentes. Además, la dotación del sector y sus características como tamaño, mezcla de recursos y grado de desarrollo definen la estructura de mercado inicial y el punto de arranque que determina la vía que seguirá la reforma y sus resultados. La estructura de mercado depende de varias medidas, como la desintegración de empresas integradas y de factores institucionales como las autoridades regulatorias y la legislación. Esta naturaleza mul- 
tifacética de la reforma produce una variedad de resultados, de tal forma que la efectividad y el impacto pueden ser medidos a través de diferentes indicadores de desempeño como la eficiencia operativa o el incremento en la inversión. ${ }^{8}$

El desempeño puede ser evaluado por medio de varias dimensiones. La propiedad privada, la competencia y la reforma regulatoria deben mejorar la eficiencia técnica reflejada en la utilización de la capacidad, la eficiencia operativa, la productividad laboral y las pérdidas de energía. En el caso de los países en desarrollo, una motivación clave para llevar a cabo una reforma es atraer capital privado para reducir la carga sobre el presupuesto público y acelerar el ritmo de inversión en nueva capacidad. Los cambios en los precios son presumiblemente el factor más esencial para los consumidores, aunque en algunos países puede ser más importante la confiabilidad. Los cambios de precios intentan medir hasta qué punto las ganancias en productividad han sido transferidas a los consumidores. Los incrementos de precios reales pueden no ser del todo indeseables, si éstos eran demasiado bajos debido al uso de subsidios.

De acuerdo con lo anterior, se seleccionaron indicadores adoptados que reflejan las etapas de reforma: privatización de la generación, separación vertical entre generación y transmisión, así como la existencia de un mercado mayorista. Este último indicador refleja también la estructura de mercado junto con el grado de desintegración vertical. El acceso de terceros a la red refleja la regulación. El desempeño técnico se mide por las pérdidas en la transmisión y distribución de electricidad, la capacidad instalada, la generación neta y los precios domésticos e industriales. Por último, se toman en cuenta indicadores macro como el consumo per cápita de electricidad y el PIB por unidad de uso de energía?

${ }^{8}$ T. Jamasb, D. Newbery, y M. Pollitt, Core Indicators for Determinants and Performance of Electricity Sector in Developing Countries, Cambridge, Cambridge Working Papers in Economics (CWPE) 0438, 2004.

${ }^{9}$ Véase el anexo para una descripción de las variables examinadas y para una reseña detallada del análisis realizado, Alfonso Brown, Las instituciones como factor competitivo: un análisis comparativo del sector eléctrico con distintos procesos de integración, Barcelona, 2008, 274 pp. (Tesis de doctorado en Relaciones Internacionales e Integración Europea por el Instituto Universitario de Estudios de la Integración Europea, por la Universidad Autónoma de Barcelona). En http://www.tesisenxarxa.net/TDX-0331109-131529/index.html. 
Se espera que una reforma del sector eléctrico, en cualquier modalidad, produzca los siguientes resultados: una mejoría en la eficiencia con que operan las empresas y que se traduzca en una disminución de las pérdidas en la transmisión y distribución de electricidad, así como una rebaja en los precios industriales. Por otra parte, debe producir también un incremento en la capacidad instalada, así como la generación neta de electricidad y el consumo per cápita.

Los mismos resultados se esperan al privatizarse la generación de electricidad y al separarse de modo vertical los segmentos de generación y transmisión, de la misma forma que al desintegrarse verticalmente la industria en su conjunto. La privatización debe ir acompañada del libre acceso de terceros a la red de transmisión, pues de lo contrario las empresas privatizadas y las nuevas entrantes al mercado podrían ser sujetas a prácticas monopólicas que les impidan competir en igualdad de condiciones. De igual forma, la existencia de un mercado mayorista es parte importante del proceso de reforma y, por lo tanto, se espera que tenga los mismos efectos sobre las variables de desempeño de la industria y las variables macroeconómicas. En cuanto a la integración y a la separación vertical, se espera que mientras más separadas o desintegradas sean las compañías, mejor sea el desempeño.

Los datos utilizados cubren el periodo 1980-2003 y se obtuvieron de varias fuentes: de la Agencia Internacional de Energía, ${ }^{10}$ de la oECD, ${ }^{11}$ del Banco Mundial, ${ }^{12}$ así como de una base de datos elaborada por Jamasb, Newbery y Pollitt. ${ }^{13}$

En una primera parte se realizó un análisis de varianza entre países. Con ello se pretende demostrar que los diferentes arreglos institucionales existentes en cada país tienen efectos diferenciados sobre las variables de desempeño ya

${ }^{10}$ International Energy Agency, Electricity Information, París, IEA Statistics, 2006.

${ }^{11}$ OECD International Regulation Database. En http://www.oecd.org/document/1/0,3343, en_2649_34323_2367297_1_1_1_1,00.html.

${ }_{12}$ World Bank (2010), World Development Indicators, Washington, D.C. En http://databank. worldbank.org/ddp/home.do.

${ }^{13}$ T. Jamasb, R. Motta, D. Newbery y M. Pollitt, "Electricity Sector Reform in Developing Countries: A Survey of Empirical Evidence on Determinants and Performance", en Cambridge Working Papers in Economics, Cambridge, CWPE 0439, 2004. 
comentadas. De este análisis se concluye que la industria eléctrica española es la que ha alcanzado un mayor grado de desarrollo, seguida de la chilena.

El análisis empírico muestra que existen diferencias significativas entre los países para prácticamente todas las variables utilizadas. En un estudio más detallado fue posible determinar, con mayor precisión, dónde radican las diferencias en el desempeño de los sectores eléctricos de estas naciones. Las pruebas muestran que hay diferencias significativas en lo que se refiere a las pérdidas de electricidad en la generación y transmisión entre todos los países. En cuanto al consumo per cápita de electricidad, hay similitudes entre Argentina, Chile y México; en cambio España contrasta claramente. Este resultado confirma la diferencia que existe en niveles de ingreso per cápita y, asociado a éste, en el consumo de electricidad, así como en el desarrollo económico. España tiene un ingreso per cápita muy superior a los otros tres países y, consecuentemente, un consumo de electricidad considerablemente mayor. En cuanto a los precios industriales la única diferencia significativa es la que hay entre México por un lado y Argentina, Chile y España por el otro.

$\mathrm{Al}$ introducirse condiciones de competencia en el mercado, se espera que desaparezcan los subsidios cruzados que suelen existir entre tarifas industriales y domésticas, abaratando las primeras y encareciendo las segundas. En lo que respecta a los precios domésticos no se encontró una diferencia sustancial entre Argentina y Chile, pero sí entre estos dos países y España, así como con México, de la misma forma que también la hay entre estos dos últimos países. Los niveles de tarifas más bajos, tanto industrial como residencial, corresponden en promedio a México, seguido por Argentina, Chile y España en ese orden. Lo anterior puede considerarse como una consecuencia del grado de liberalización del sector eléctrico en dichos países, y de que las tarifas eléctricas guardan mayor proporción con los costos marginales de suministro.

Con relación al grado de eficiencia de la industria, medido como el porcentaje de pérdidas en la transmisión y distribución de electricidad, cada país pertenece a una categoría diferente que puede interpretarse como el grado de eficiencia de sus respectivos mercados, siendo el más eficiente el español, seguido por el chileno, el mexicano y el argentino. España y Chile son los países 
que muestran mejor desempeño, tal como puede observarse en la Gráfica 3. Esto corrobora el hecho de que estos dos países también han sido los que más han avanzado en el proceso de reforma y liberalización de su sector eléctrico.

\section{Gráfica 3}

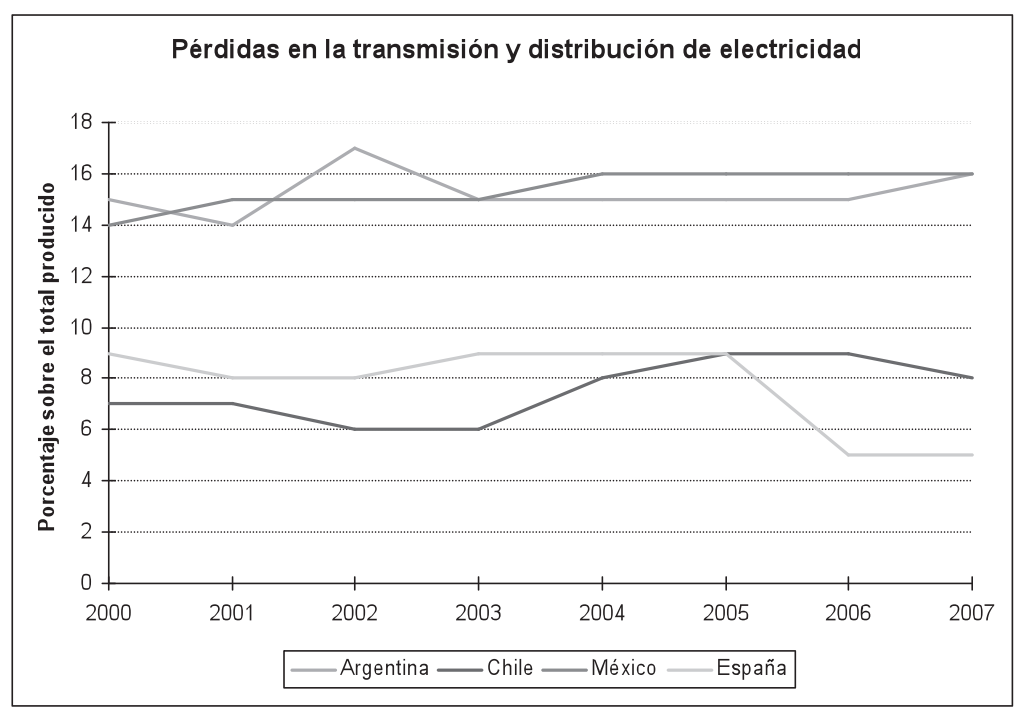

Elaboración propia con base en información del Banco Mundial, World Development Indicators.

En cuanto al consumo per cápita, se confirma que existe una clara diferencia entre España y los demás países, como puede apreciarse en la Gráfica 4. Esta variable se encuentra muy correlacionada con el nivel de desarrollo económico. Lo mismo se puede observar en la siguiente gráfica en la que el consumo per cápita más bajo le corresponde a México, muy por debajo del de España. 
Gráfica 4

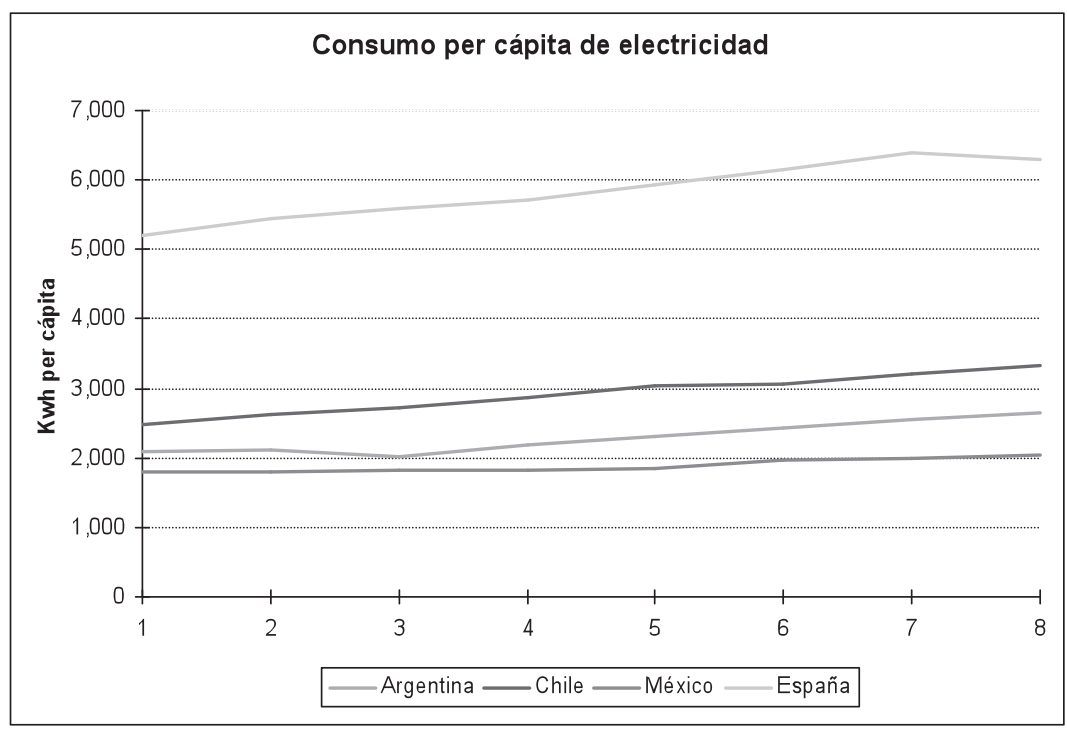

Elaboración propia con base en información del Banco Mundial, World Development Indicators.

En cuanto a precios industriales (véase la Gráfica 5), México se diferencia de los demás países, ya que durante la mayor parte del periodo analizado tuvo niveles inferiores a los demás como puede apreciarse en la gráfica correspondiente. Esto puede explicarse porque buena parte de la generación de electricidad se hizo a partir de hidrocarburos como fuente primaria de energía y el precio de los mismos mantuvo considerables subsidios durante dicho periodo. Pero, además, es necesario tener precaución con este indicador, así como con el de precios domésticos, pues la medición está hecha en dólares norteamericanos corrientes y, como se sabe, éstos resultan afectados por el tipo de cambio. Además, son precios con impuestos incluidos y las tasas impositivas son distintas en cada país. Más que una simple comparación directa, resulta de mayor interés observar la tendencia de dichos precios en cada país durante el periodo de análisis. Así, se aprecia que en España los precios registraron una tendencia descendente desde 1992 
hasta 2001, periodo que coincide con la liberalización del sector eléctrico, primero con la Ley de Ordenación del Sistema Eléctrico (LOSEN) en 1994 y después en 1997 con la Ley del Servicio Eléctrico (LSE). En el caso de México la entrada en vigor del TLCAN no parece haber tenido un efecto favorable sobre los precios, pues desde entonces éstos registraron una tendencia ascendente. Esto puede explicarse por la falta de apertura e integración del mercado mexicano con el de sus socios comerciales y la ausencia de instituciones supranacionales que obliguen a introducir mecanismos competitivos en el área de integración.

\section{Gráfica 5}

\section{Precios industriales}

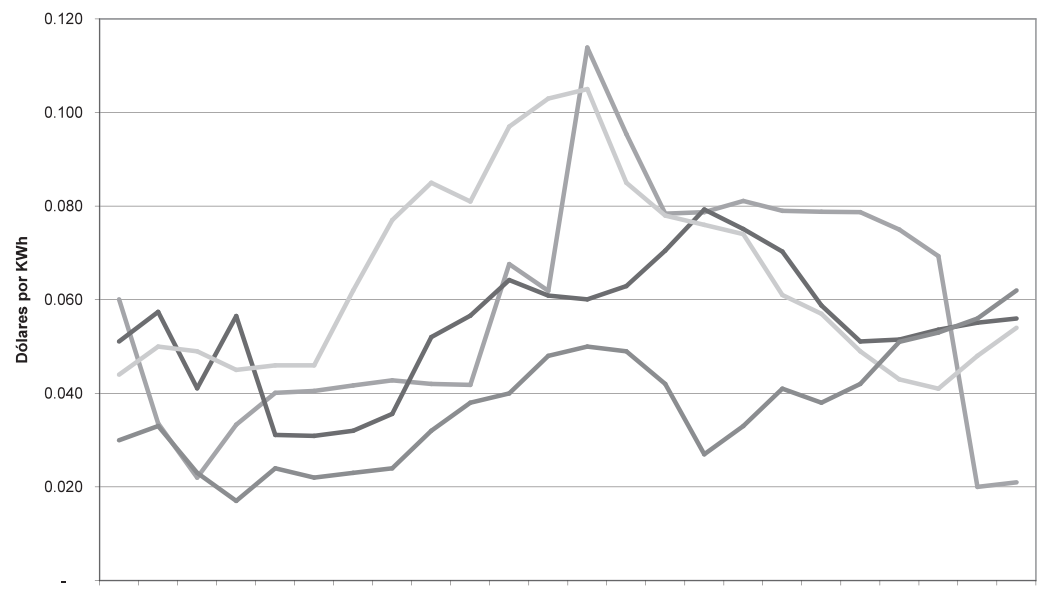

198019811982198319841985198619871988198919901991199219931994199519961997199819992000200120022003

$$
\text { - Argentina - Chile - España - México }
$$

Elaboración propia con base en información de la Agencia Internacional de Energía (AIE).

Respecto a los precios domésticos (véase la Gráfica 6), se observa un comportamiento muy similar al de los precios industriales, tres grupos: México, Argentina y Chile por un lado, y España por el otro. Sin embargo, hay que tomar 
este resultado con cautela pues los precios domésticos en México recibieron subsidios que fluctuaron entre 50 y $70 \%$ en dicho periodo. ${ }^{14}$

\section{Gráfica 6}

\section{Precios residenciales}

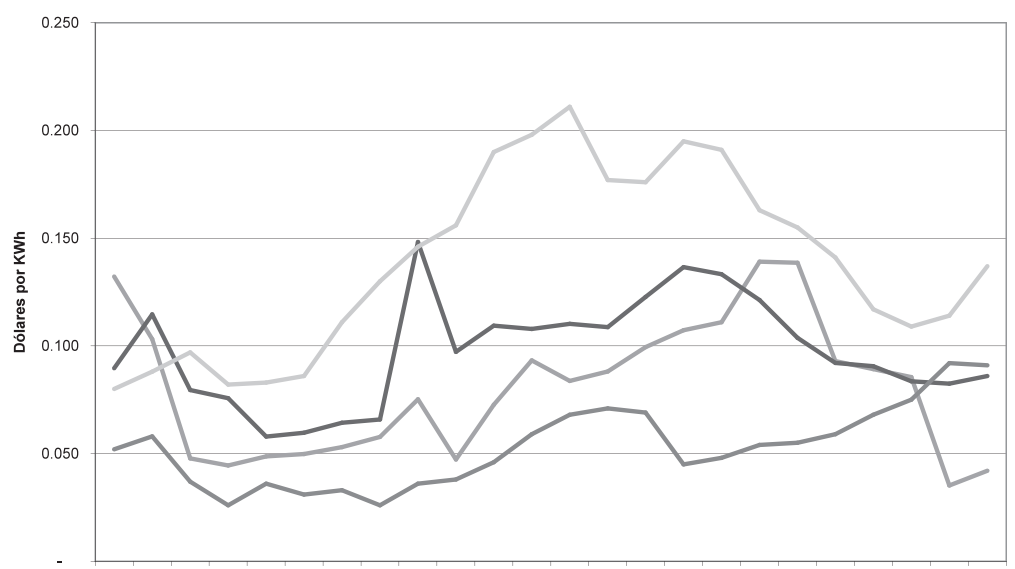

198019811982198319841985198619871988198919901991199219931994199519961997199819992000200120022003 - Argentina - Chile España - México

Elaboración propia con base en información de la Agencia Internacional de Energía (AIE).

Puede concluirse del análisis anterior que existen diferencias significativas entre los países estudiados y que tales diferencias responden a los factores examinados.

El propósito de la segunda parte del análisis fue determinar el efecto que tuvo en cada país la introducción de ciertas reformas en la organización y el funcionamiento de sus mercados eléctricos. La hipótesis general es que a partir de la introducción de diversas reformas mejora el desempeño de la industria en cada país. En un mercado competitivo los precios y las utilidades revelan infor-

${ }^{14}$ Véase César Hernández, La reforma cautiva. Inversión, trabajo y empresa en el sector eléctrico mexicano, México, Centro de Investigación para el Desarrollo, A. C., 2007, p. 476. 
mación importante acerca de los costos y la eficiencia con que se emplean los insumos, al mismo tiempo que proporcionan incentivos para que las empresas se esfuercen por mejorar su eficiencia interna. Por ello es de esperarse que la competencia propicie menores costos unitarios, producto de una creciente eficiencia técnica, que puede ser traspasada a los consumidores mediante menores precios, y estimular con ello un incremento en la cantidad demandada. Por lo tanto, se espera que la competencia tenga efectos positivos tanto sobre la oferta como sobre la demanda de electricidad.

\section{HIPÓTESIS SOBRE COMPETENCIA}

La introducción de esquemas competitivos puede impactar los precios de diferente manera dependiendo del tipo de consumidor. La competencia disminuye la posibilidad de que existan subsidios cruzados de un tipo de consumidores hacia otros porque, en caso de haberlos, las empresas entrantes se enfocarían hacia los consumidores con sobreprecio y con ello provocarían una reducción en éste. Dado que generalmente existen subsidios cruzados cuando la propiedad es estatal, se espera que la competencia reduzca los precios industriales o la relación entre los mismos, y los precios de los consumidores domésticos. Habrá una expectativa de que los precios domésticos aumenten, aunque esto no es del todo seguro debido a que la competencia puede resultar en menores costos de producción y, por lo tanto, menores precios para usuarios residenciales, aun cuando se eliminen los subsidios cruzados. ${ }^{15}$

En general, los resultados esperados con la introducción de algún grado de reforma o liberalización del mercado son:

- que disminuyan las pérdidas de transmisión y distribución de electricidad, - que aumente la capacidad instalada,

\footnotetext{
${ }^{15}$ Véase Y-F Zhang, C. Kirkpatrick, y D. Parker, "Electricity Sector Reform in Developing Countries: An Econometric Assessment of the Effects of Privatization, Competition and Regulation", en Discussion Paper, núm. 31, Centre on Regulation and Competition, Institute for Development Policy and Management, Manchester, University of Manchester, 2002.
} 
- que se incremente la generación neta de electricidad,

- que aumente el consumo per cápita,

- que disminuyan los precios industriales y

- que disminuya la relación precios domésticos/precios industriales.

El acceso de terceros a la red de transmisión y la existencia de un mercado mayorista son etapas progresivas en el proceso de reforma y, por lo tanto, se espera que también impacten favorablemente sobre las variables de desempeño de la industria.

En términos generales se esperan resultados positivos en la medida que se privatice la actividad de generación. La nueva economía institucional proporciona importantes hipótesis sobre los efectos de diferentes tipos de estructuras de propiedad sobre los incentivos. ${ }^{16}$ Se espera que la privatización eleve la eficiencia económica mediante 1) una modificación en la asignación de los derechos de propiedad, que implica una diferente estructura de incentivos administrativos y de conducta directiva; 2) una eliminación del "presupuesto blando" financiado a través de los contribuyentes, y una exposición de las empresas a la disciplina del mercado de capital, con lo cual se introducen objetivos más precisos y medibles que reducen los costos de transacción, en especial los asociados con el monitoreo de los agentes por parte de los principales; y 3) una eliminación de la interferencia política en la operación de las empresas, que evita su captura por grupos especiales de interés.

Es de esperarse que la privatización induzca una mayor utilización del acervo de capital (mayor utilización de la capacidad). Sin embargo, cuando se aplican estos principios teóricos a algunos aspectos del desempeño en el sector eléctrico, se deben tener presentes algunas características específicas de dicha actividad. La industria eléctrica se caracteriza por grandes inversiones hundidas, escalas mínimas de operación y un producto no almacenable de consumo masivo, que presenta el riesgo de un comportamiento oportunista que puede afec-

\footnotetext{
${ }^{16}$ Véase Douglass North, Instituciones, cambio institucional y desempeño económico, México, FCE, 1993, 190 pp.; así como B. Levy y P. T. Spiller [eds.] Regulations, Institutions and Commitment, Cambridge, Cambridge University Press, 1996.
} 
tar los intereses de los inversionistas. Al saber que en ciertas circunstancias los gobiernos son incapaces de cumplir algunos acuerdos explícitos o implícitos y comportarse oportunistamente, los inversionistas privados pueden mostrarse reacios a invertir en la expansión de la capacidad instalada. Esto puede hacer que el verdadero efecto de la privatización sobre el crecimiento de la capacidad de generación y de utilización no sea del todo claro, a pesar de que uno de los principales propósitos de los gobiernos al privatizar es que haya mayor inversión de capital en la infraestructura eléctrica.

Uno de los componentes en la función y objetivo de las empresas eléctricas, propiedad del Estado, es proveer servicios básicos a tantos individuos como sea posible, incluso a precios por debajo de los costos incurridos, en particular en el caso de los consumidores residenciales. En contraste, cuando las empresas propiedad del Estado son transferidas a manos privadas es muy probable que los nuevos propietarios sean reacios a incrementar la cantidad de electricidad generada a menos que sea rentable hacerlo, con lo cual los precios se establecen en función de los costos marginales.

Históricamente los precios de la electricidad han sido fijados por los gobiernos de forma ineficiente (no se relacionan con los costos marginales), con el propósito de alcanzar la mayor cobertura posible y con base, muchas veces, en criterios clientelares dado el peso político de los usuarios individuales de electricidad en las votaciones. Por eso es que los usuarios domésticos con frecuencia reciben subsidios cruzados de otros tipos de usuarios, en especial industriales. La privatización puede inducir, entonces, mayores precios para los consumidores residenciales y menores precios para los industriales, dado que los precios están alineados con los costos de largo plazo y debido a que las utilidades, no los votos, son la preocupación fundamental de los inversionistas privados.

En cuanto a la integración y a la separación vertical, se espera que mientras más separadas o desintegradas sean las compañías, mejor sea el desempeño de la industria eléctrica, dado que la privatización del segmento de generación implica la desintegración vertical de la industria y con ello promueve la competencia.

La existencia de un proceso de reforma explica por sí sola la mayor parte de las diferencias entre los países, seguida de la existencia de un mercado mayorista 
de electricidad y de la separación vertical entre los segmentos que componen la industria. En cambio, la forma de propiedad no mostró ser un factor tan importante como tampoco el grado de integración entre compañías.

\section{HIPÓTESIS SOBRE EL TIPO DE PROPIEDAD}

Un análisis detallado entre la estructura de propiedad y el comportamiento de variables de desempeño ayuda a entender, con mayor precisión, el origen de las diferencias entre grupos (véanse los resultados en el anexo). Así, se aprecia que el efecto más importante del tipo de propiedad es aquél que se da con relación a las pérdidas de transmisión y distribución de electricidad: entre, por un lado, la propiedad pública y, por otro, cualquier forma de propiedad que incluya algún grado de participación privada. La propiedad pública está asociada con mayores pérdidas de electricidad. Y éstas disminuyen conforme aumenta la participación privada.

En cuanto a la relación entre el tipo de propiedad y la capacidad instalada, se aprecian diferencias importantes en lo que se refiere a la propiedad mayoritariamente pública (México) y la propiedad privada. Resulta interesante esta diferencia, pues la media del primer factor antes señalado muestra una mayor capacidad instalada que en los demás, lo cual confirmaría la hipótesis de que las empresas públicas tienen mayor propensión a invertir en elefantes blancos. Esto debe tomarse con precaución pues se trata de datos brutos que habría que contextualizar, ya que al final del periodo analizado la inversión en capacidad instalada en México se rezagó con relación al crecimiento en la demanda debido a restricciones de carácter presupuestal.

Con relación a la generación neta, hay diferencias notables entre la propiedad mayoritariamente pública y la totalmente pública por un lado, con la propiedad privada por el otro. Esto hace pensar que la estructura de propiedad efectivamente repercute sobre la generación de electricidad, aunque tampoco se puede asegurar con certeza.

En términos de precios industriales, hay diferencias claras entre todos los tipos de propiedad y la pública, tal como puede observarse de acuerdo con el nivel de significancia. En el caso de los precios domésticos las diferencias están presentes en todos los tipos de propiedad. Esto debe tomarse con cautela pues los precios 
están expresados en dólares y, por lo tanto, están en función del tipo de cambio. Las empresas públicas suelen subsidiar las tarifas eléctricas, particularmente las domésticas. Sin embargo, también es cierto que estos subsidios sirven para cubrir la ineficiencia de la propia industria, debido a la falta de competencia, a que la selección de las tecnologías empleadas se basa en precios relativos distorsionados por subsidios públicos, que con el tiempo son imposibles de sostener, así como la captura de las empresas públicas por parte de grupos de interés. Tal es el caso de México, en el que los precios de los derivados del petróleo fueron subsidiados por mucho tiempo y en que los sindicatos han extraído sustanciales rentas, y se han organizado exitosamente para bloquear la liberalización de la industria.

\section{HIPÓTESIS SOBRE LA INTEGRACIÓN VERTICAL}

El análisis de la interacción entre los distintos grados de integración vertical de la industria, y las demás variables muestra que hay diferencias significativas en lo que se refiere al efecto de dicha variable sobre las pérdidas en la transmisión y distribución de electricidad. En términos de capacidad instalada y generación de electricidad también existen diferencias significativas entre un mercado integrado y otro desintegrado. Los resultados pueden verse en el anexo.

\section{HIPÓTESIS SOBRE EL GRADO DE INTEGRACIÓN ENTRE EMPRESAS DE GENERACIÓN Y TRANSMISIÓN}

En cuanto al grado o tipo de separación entre empresas, se observan efectos importantes sobre las pérdidas en la transmisión y distribución, pero no representativos respecto a la generación de electricidad. Los resultados completos pueden verse en el anexo.

Más importante todavía es que la separación entre compañías tiene repercusiones sobre los precios industriales y domésticos. 


\section{CONCLUSIONES}

A pesar de que el proceso de integración de América Latina tuvo un impulso significativo en las dos últimas décadas, subsisten numerosos obstáculos que impiden un vínculo más estrecho no sólo en el comercio regional, sino en la integración de los mercados y la competencia. El sector eléctrico es una de las áreas en las que la combinación de reformas e integración propicia un ambiente más competitivo en beneficio de toda la actividad económica. La experiencia europea en este terreno muestra que para que ello pueda avanzar se requiere la creación de instituciones comunes que no sólo establezcan el camino a seguir, sino que tengan facultades por encima de los gobiernos nacionales para eliminar los obstáculos que surgen en el proceso. En particular, la creación de un mercado competitivo en el que las industrias eléctricas de distintos países se puedan integrar operando en un mismo mercado.

Del análisis empírico realizado se concluye, en primer término, que los factores institucionales considerados muestran efectos significativos sobre las medidas de desempeño de la industria eléctrica. En particular indican un importante poder explicativo sobre las pérdidas en la transmisión y distribución de electricidad variable, utilizada para medir el grado de eficiencia en la operación.

La variable reforma incluye todos los elementos que componen el proceso completo de liberalización de la industria eléctrica, desde la creación de una nueva legislación donde tenga cabida la inversión privada, aun con márgenes estrechos de operación, pasando por la introducción de elementos regulatorios independientes, como en el caso de México, hasta liberalización del comercio de electricidad para que se negocie a través de mercados organizados o en forma bilateral, como en los casos de Argentina, Chile y España. En cualquiera de sus grados de avance, dicha variable explica por sí sola la mayor parte de las diferencias en el desempeño de los países analizados, y con ellos mismos antes de introducir reformas. La existencia de un mercado mayorista y la separación vertical entre los segmentos que componen la industria son también elementos importantes de las reformas competitivas. El tipo de propiedad produce efectos positivos sobre la eficiencia operativa. La privatización de la generación, acompañada de la desintegración de la industria, provoca menores pérdidas en 
la transmisión y distribución, así como un mayor consumo per cápita. Sin embargo, esta mejora en la eficiencia no se traduce necesariamente en una mejor estructura de precios. Esto se explica por las distorsiones que introduce la regulación, así como por sus omisiones.

También la desintegración vertical entre generación y transmisión, así como la separación entre compañías tienen efectos significativos sobre las pérdidas de transmisión y distribución y el consumo per cápita. La desintegración vertical no muestra efectos significativos en todos los casos sobre los precios, pero la separación entre compañías sí los tiene.

De lo anterior se deduce que para que la privatización de la generación de electricidad rinda los frutos esperados hace falta acompañarla de una adecuada regulación, que haga efectiva la desintegración vertical y la separación entre compañías. La existencia de normas antimonopolio de carácter supranacional y la libre circulación de capitales, fruto del proceso de integración, aseguran a largo plazo la integración del sector eléctrico en un marco de integración económica.

Recibido: 29 de abril, 2010. Aceptado: 10 de junio, 2010.

\section{BiBLIOGRAFÍA}

BRown, AlFonso, Las instituciones como factor competitivo: un análisis comparativo del sector eléctrico con distintos procesos de integración, Barcelona, 2008, 274 pp. (Tesis de doctorado en Relaciones Internacionales e Integración Europea, Instituto Universitario de Estudios de la Integración Europea, Universidad Autónoma de Barcelona). En http://www.tesisenxarxa. net/TDX-0331109-131529/index.html.

CEPAL, OLADE, GTZ, "Energía y desarrollo sustentable en América Latina y el Caribe: guía para la formulación de políticas energéticas", en Cuadernos de la CEPAL, núm. 89, Santiago de Chile, diciembre de 2003. 
Costas, Antón, "Economía política de la regulación y la desregulación en España", en J. C. Jiménez [ed.], La economía española ante el nuevo siglo, Madrid, Cívitas, 2001, pp. 39-64.

Fernández Ordóñez, Miguel Ángel, La competencia, Madrid, Alianza Editorial, 2000.

HERNÁNDEZ, CÉSAR, La reforma cautiva. Inversión, trabajo y empresa en el sector eléctrico mexicano, México, Centro de Investigación para el Desarrollo, A.C., 2007.

International ENergy Agency, Electricity Information, París, IEA Statistics, 2006.

Jamasb, T., Newbery, D., y M. PoluitT, "Core Indicators for Determinants and Performance of Electricity Sector in Developing Countries", en Cambridge Working Papers in Economics, Cambridge, CWPE 0438, 2004.

Jamasb, T., R. MotTa, D. Newbery, y M. PoluitT "Electricity Sector Reform in Developing Countries: A Survey of Empirical Evidence on Determinants and Performance", en Cambridge Working Papers in Economics, Cambridge, CWPE 0439, 2004.

Levy, B. y P. T. Spiller [eds.] Regulations, Institutions and Commitment, Cambridge, Massachusetts, Cambridge University Press, 1996.

NorTH, Douglass C., Instituciones, cambio institucional y desempeño económico, México, FCE, 1993.

OECD International Regulation Database. En http://www.oecd.org/document/1/0,3343,en_2649_34323_2367297_1_1_1_1,00.html.

Olson, Mancur, The Logic of Collective Action: Public Goods an the Theory of Groups, Cambridge, Massachusetts, Harvard University Press, 1965.

, The Rise and Decline of Nations: Economic Growth, Stagflation and Social Rigidities, New Haven, Yale University Press, 1982.

SÁEz, SeBAStí́n, La integración en busca de un modelo: los problemas de convergencia en América Latina y el Caribe, CEPAL, Serie Comercio Internacional, 88, Santiago de Chile, 2008. 
Stigler, George, "The Theory of Economic Regulation", en Bell's Journal of Economics and Management Science, Spring, 1971, pp. 3-21.

World Bank (2010). World Development Indicators. Washington, D.C. En http://databank.worldbank.org/ddp/home.do.

Zhang, Y-F., C. KirKPatrick, y D. PARKer, "Electricity Sector Reform in Developing Countries: An Econometric Assessment of the Effects of Privatization, Competition and Regulation", en Discussion Paper, núm. 31, Manchester, Centre on Regulation and Competition, Institute for Development Policy and Management, University of Manchester, 2002.

ANEXO

\begin{tabular}{|c|c|c|c|c|}
\hline \multicolumn{3}{|c|}{ Variables independientes (institucionales) } & \multicolumn{2}{|c|}{ Variables dependientes (desempeño) } \\
\hline Nombre & Descripción & Valores & Nombre & Descripción \\
\hline Reforma & $\begin{array}{l}\text { Existencia de } \\
\text { reforma } \\
\text { eléctrica }\end{array}$ & $\begin{array}{l}0=\text { no } \\
1=\text { sí }\end{array}$ & translos & $\begin{array}{l}\text { Pérdidas de electricidad en } \\
\text { transmisión y distribución }\end{array}$ \\
\hline Tpa & $\begin{array}{l}\text { Acceso de } \\
\text { terceros a la } \\
\text { red de } \\
\text { transmisión }\end{array}$ & $\begin{array}{l}0=\text { no } \\
1=\text { sí }\end{array}$ & capinst & Capacidad instalada total \\
\hline Propiedad & $\begin{array}{l}\text { Tipo de } \\
\text { propiedad }\end{array}$ & $\begin{array}{l}0=\text { pública } \\
1=\text { mayoritariamente } \\
\text { pública } \\
2=\text { mayoritariamente } \\
\text { privada } \\
3=\text { privada }\end{array}$ & gener & Generación total neta \\
\hline Mayorías & $\begin{array}{l}\text { Existencia de } \\
\text { mercado } \\
\text { mayorista }\end{array}$ & $\begin{array}{l}0=\text { no } \\
1=\text { sí }\end{array}$ & conspc & Consumo per cápita \\
\hline vertint & $\begin{array}{l}\text { Integración } \\
\text { vertical entre } \\
\text { generación y } \\
\text { transmisión }\end{array}$ & $\begin{array}{l}0=\text { integrado } \\
1=\text { separación contable } \\
2=\text { compañías } \\
\text { separadas }\end{array}$ & precind & $\begin{array}{l}\text { Precios de la electricidad } \\
\text { para la industria en } \\
\text { dls } / \mathrm{kWh}\end{array}$ \\
\hline sepvert & $\begin{array}{l}\text { Grado de } \\
\text { separación } \\
\text { vertical de la } \\
\text { industria en } \\
\text { general }\end{array}$ & $\begin{array}{l}0=\text { integrado } \\
1=\text { mixta } \\
2=\text { desintegrado }\end{array}$ & precdom & $\begin{array}{l}\text { Precios de la electricidad } \\
\text { para los hogares en } \\
\text { dls } / \mathrm{kWh}\end{array}$ \\
\hline & & & pibpc & PIB per cápita \\
\hline & & & pibpue & $\begin{array}{l}\text { PIB por unidad de uso de } \\
\text { energía }\end{array}$ \\
\hline
\end{tabular}

